\title{
The Burnside Groups and Small Cancellation Theory
}

\author{
Jonathan P. McCammond
}

December 23, 2017

\begin{abstract}
In a pair of recent articles円, the author develops a general version of small cancellation theory applicable in higher dimensions ([5]), and then applies this theory to the Burnside groups of sufficiently large exponent ([6]). More specifically, these articles prove that the free Burnside groups of exponent $n \geq 1260$ are infinite groups which have a decidable word problem. The structure of the finite subgroups of the free Burnside groups is calculated from the automorphism groups of the general relators used in the presentation. The present article gives a brief introduction to the methods and techniques involved in the proof. Many of the ideas originate with the recent work of A. Yu. Ol'shanskii and S. V. Ivanov. Some familiarity with their work on the Burnside problem will be assumed.
\end{abstract}

\section{Introduction}

Ninety-five years ago William Burnside asked whether every finitely generated group of finite exponent must be finite [2]. Since then, significant progress has been made in the study of the free Burnside groups, much of it coming in recent years. In this first section the recent history of their study will be briefly reviewed so that the results announced below can be

\footnotetext{
${ }^{1}$ As of 1 Dec 1997, one article has been accepted for publication and the second will soon be available as a preprint. Until the second article has been distributed, all of the results which refer to the Burnside groups in particular should be considered preliminary announcements only. The exponent of 1260 is especially provisional.
} 
viewed in their proper context. The second section introduces many of the concepts from which the general theory is constructed. The third and the fourth sections will describe the results of general small cancellation theory and the results on the Burnside groups in greater detail.

\section{$1.1 \quad$ Historical Context}

The existence of exponents for which Burnside's conjecture is false was first demonstrated by Novikov and Adian in a series of articles published in 1968 [7]. More specifically, for $n \geq 4381$ and odd, they proved that the free Burnside group of exponent $n$ is infinite, that these groups have decidable word problems, and that their only finite subgroups are cyclic. In a book-length reworking of the results [1], Adian was able to extend coverage of the proof to include all cases with $n \geq 665$ and odd. Both versions of the proof are several hundred pages in length.

In 1982, A. Yu. Ol'shanskii published an alternative proof that the Burnside groups of exponent $n>10^{10}$ and odd are infinite, that they have a decidable word problem, and that their only finite subgroups are cyclic [8]. Ol'shanskii used small cancellation theory and especially van Kampen diagrams to create a significantly shorter proof of Adian and Novikov's results. By introducing more geometry, the proof was reduced to a mere thirty-page article.

In 1994, S. V. Ivanov published a substantial article on the Burnside groups in which he showed that when $n$ is at least $2^{48}$ and either odd or divisible by $2^{9}$, then the free Burnside group of exponent $n$ is infinite [3]. In addition, he showed that these groups have a decidable word problem and he effectively described their finite subgroups. Since every Burnside group with exponent at least $2^{57}$ has one of these groups as a homomorphic image, Ivanov's proof shows that Burnside's original conjecture can be answered in the negative for almost all exponents $n$.

Finally, in 1996 I. Lysionok published a proof that the Burnside groups of exponent $n=16 k \geq 8000$ are infinite [四]. Combined with the earlier proofs regarding Burnside groups of large odd exponent, this work also shows that almost all of the Burnside groups are infinite.

As can be seen from the above review, the study of an arbitrary Burnside group of large exponent has proceeded indirectly through the study of the homomorphic images onto Burnside groups whose exponent is either large and odd or divisible by a large power of 2 . In particular, the restriction 
that $n$ be either large and odd or divisible by a large power of 2 has always been needed to show the decidability of the word problem, the structure of the finite subgroups, and other detailed structural information. The results described below remove these restrictions.

\subsection{Announced Results}

The author is currently in the process of publishing and preparing to publish a pair of articles which first develop a general version of small cancellation theory applicable in higher dimensions ([5]), and then apply this theory to the Burnside groups of sufficiently large exponent ([6]). In combination these articles prove that the free Burnside groups of exponent $n \geq 1260$ are infinite groups which have a decidable word problem. The structure of the finite subgroups of these groups is calculated from the automorphism groups of the general relators used in the presentation. The present article gives a brief introduction to the methods and techniques involved in the proof. The definition of a general relator, the axioms of general small cancellation theory, and a description of the inductive construction involved for the Burnside groups are included. Many of the ideas involved originate with the recent work of A. Yu. Ol'shanskii and S. V. Ivanov. Some familiarity with their work on the Burnside problem will be assumed.

The theory developed by the author in [5] is a generalized version of small cancellation theory which is applicable to specific types of high-dimensional simplicial complexes. The usual results on small cancellation groups are shown to hold in this new setting with only slight modifications. The major results of this theory are summarized below in Theorem A. The definitions of the unfamiliar terms will be given later in the article.

Theorem A If $G=\langle A \mid \mathcal{R}\rangle$ is a general small cancellation presentation with $\alpha \leq \frac{1}{12}$, then the word and conjugacy problems for $G$ are decidable, the Cayley graph is constructible, the Cayley category of the presentation is contractible, and $G$ is the direct limit of hyperbolic groups. If the presentation satisfies a few additional hypotheses, then every finite subgroup of $G$ is a subgroup of the automorphism group of some general relator in $\mathcal{R}$.

The generalized small cancellation theory developed in [5] overlaps significantly with the general theories developed by Rips ([10]) and Ol'shanskii ([8]). In fact, the theory described below attempts to provide an underly- 
ing geometric object whose existence explains the success of these earlier constructions.

The main results on the Burnside groups which are proved in [6] are summarized below in Theorem $\mathbb{B}$. The notation $\mathcal{B}(m, n)$ refers to the $m$ generated free Burnside group of exponent $n$.

Theorem B For any $m>1$, and any $n \geq 1260$, the Burnside group $\mathcal{B}(m, n)$ possesses a general small cancellation presentation which satisfies all of the hypotheses and conclusions of Theorem A. In addition, every finite subgroup of the group $\mathcal{B}(m, n)$ is contained in a direct product of a dihedral of order $2 n$ with a finite number of dihedral 2-groups whose exponent divides $n$. As a consequence, all of the groups $\mathcal{B}(m, n)$ are infinite.

The approach used in these articles has succeeded in significantly lowering the previous bounds beyond which all of the Burnside groups are known to be infinite, and also in proving for the first time that the word problem for all Burnside groups of sufficiently large exponent is decidable. Finally, in contrast with earlier work on the Burnside groups, no distinction is made between Burnside groups with even or odd exponents.

\section{Preliminaries}

The generalization of small cancellation theory alluded to above will depend on an expansion of the usual definition of a relator. To help motivate the revised definition, we will begin with a few familiar examples, followed by the technical definitions.

\subsection{First Examples}

The following examples are groups with familiar presentations and wellknown properties which can be subsumed under the rubric of general small cancellation theory. The explanation of the exact definitions will be postponed until after the examples have been described.

Example 2.1 [The Modular Group] Let

$$
S=\left(\begin{array}{cc}
0 & -1 \\
1 & 0
\end{array}\right) \text { and } T=\left(\begin{array}{ll}
1 & 1 \\
0 & 1
\end{array}\right)
$$



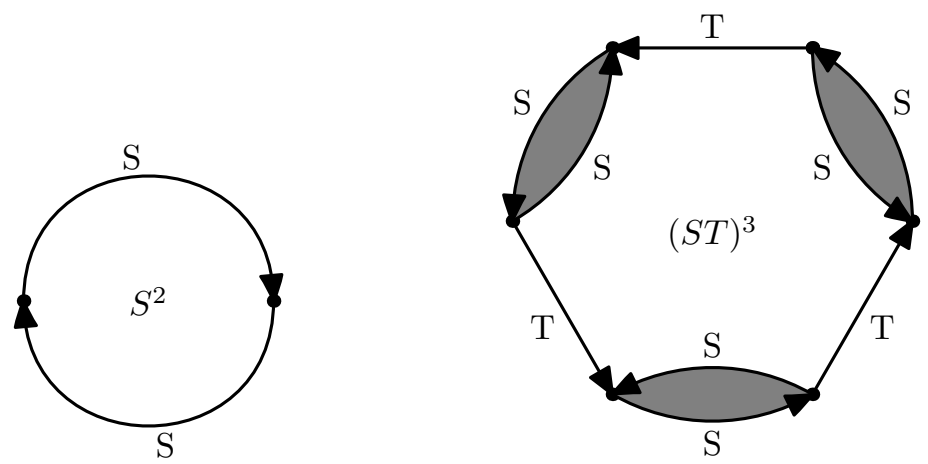

Figure 1: General relators for the modular group

Then $S^{2}=-I,(S T)^{3}=-I$. The modular group $\operatorname{PSL}_{2}(\mathbb{Z})=\mathrm{SL}_{2}(\mathbb{Z}) / \pm 1$ is the free product of the cyclic groups of order 2 and 3 which are generated by $S$ and $S T$. In particular, $\mathrm{PSL}_{2}(\mathbb{Z})$ is generated by $S$ and $T$, and it has the following presentation:

$$
\mathrm{PSL}_{2}(\mathbb{Z})=\left\langle S, T \mid S^{2}=(S T)^{3}=1\right\rangle
$$

This particular presentation does not satisfy any of the usual small cancellation conditions such as $C(6)$ or $C^{\prime}\left(\frac{1}{6}\right)$ since the letter $S$ is a piece and it represents one-half of the boundary of the first relator. The general small cancellation theory under discussion would replace this presentation with a 'general presentation' which still has $S$ and $T$ as generators, but the relators will be altered to appear as in Figure 1.

Example 2.2 [Dihedral Groups] Consider the standard presentation of the dihedral group of order $2 n$ :

$$
\mathrm{D}_{2 n}=\left\langle a, b \mid a^{2}=b^{2}=(a b)^{n}=1\right\rangle
$$

This presentation also has a modification which satisfies some general small cancellation conditions. The resulting general relators are given in Figure 8 Moreover, it is easy to show that no presentation of a dihedral is ever a small cancellation presentation in the traditional sense, since one of the results of the traditional theory is that all of the finite subgroups of a small cancellation group are cyclic. 

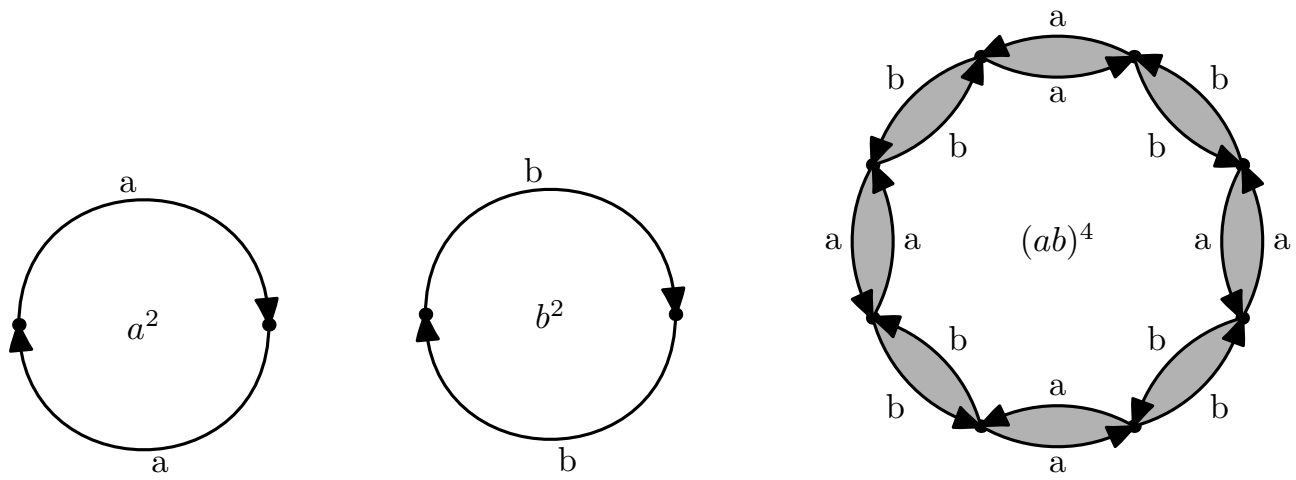

Figure 2: General relators for the dihedral group of order 8

Example 2.3 [Coxeter Groups] Coxeter groups can be seen as a generalization of Example 2.2. Let $M$ be a symmetric $n \times n$ matrix whose entries lie in the set $\mathbb{Z}^{+} \cup \infty$. Assume in addition that $m_{i i}=1$ and $m_{i j}>1$ for all $i \neq j$. The Coxeter group based on $M$ is given by the presentation

$$
G=\left\langle a_{1}, a_{2}, \ldots, a_{n} \mid\left(a_{i} a_{j}\right)^{m_{i j}}=1\right\rangle
$$

where no relation is added in the case $m_{i j}=\infty$. If $m_{i j}$ is never equal to 2 , then the Coxeter group is said to be of large type. If $m_{i j}$ is never equal to 2 or 3 , then the Coxeter group is said to be of extra-large type. Once the relators $\left(a_{i} a_{j}\right)^{m_{i j}}$ are modified as in Example 2.2, the resulting Coxeter group satisfies a version of $C(4)$, a Coxeter group of large type satisfies a version of $C(6)$, and a Coxeter group of extra-large type satisfies a version of $C(8)$.

\subsection{General Relators}

The most important concept involved in general small cancellation theory is that of a general relator. Every general relator will be assigned a height. Traditional relators are examples of height 2 . The examples given in the previous section are general relators of height 3. Before the precise technical definition is given, the examples from the previous section will be discussed in greater detail, and we will present a single example of a general relator of height 4 . 


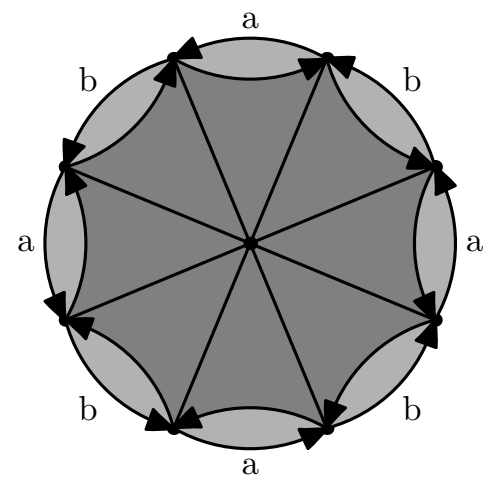

Figure 3: A more precise picture of a general relator

Definition 2.4 [Boundaries of General Relators] In the same way that a relator in the traditional sense is often viewed as a labeled disk rather than a labeled circle, the structures shown in Figure 1 and Figure 2 are, technically speaking, merely the boundaries of general relators and not the general relators themselves. A general relator will be the topological cone over its roughly circular boundary. Thus, the general relator corresponding to $(a b)^{4}$ (whose boundary is shown in Figure 2) will be composed of eight solid circular cones which are adjoined along their lateral sides. This particular general relator is shown schematically in Figure 3. Similarly the general relator corresponding to $(S T)^{3}$ (whose boundary is shown in Figure 1) will consist of three triangles and three solid circular cones.

Definition 2.5 [Heights] In a traditional relator, the vertices, the open edges and the interior of the disk can be thought of as being represented by their barycenters. Moreover, these barycenters can be assigned heights based on whether they come from a vertex (height 0 ), an edge (height 1), or a disk (height 2). In a similar way, the apex of the cone over the dihedral structure in Figure 3 can be assigned a height of 3 since it contains a disk of height 2 in its boundary. Once these barycenters have been assigned heights, there is a notion of a 1-, 2-, and 3-skeleton which is the union of all of the pieces assigned a height of at most 1 , at most 2 , or at most 3 . The rightmost picture in Figure 2 is thus the 2-skeleton of the general relator, as well as its boundary. 
Example 2.6 [A General Relator of Height 4] The following explicit example will be used to illustrate several key concepts which are precisely defined below. Let $G=\left\langle a, b, c \mid a^{2}, b^{2},(a b)^{4},(a b c b)^{2}\right\rangle$. A modification of this presentation which uses general relators begins with the three (general) relators whose boundaries are shown in Figure 2. The fourth relator, $(a b c b)^{2}$, is modified as shown in Figure 1 . It is obtained from the cycle labeled $(a b c b)^{2}$ by attaching the general relator for $(a b)^{4}$ to each of the paths labeled $b a b$. The top figure shows the 2-skeleton of this relator, while the bottom figure shows its 3-skeleton. As described above, the general relator itself will be the topological cone over this roughly circular boundary, and the apex of the cone will be assigned a height of 4 since it contains a point of height 3 in its boundary.

Examples of general relators of higher height can be obtained as follows: Given a general relator $R$ of height $k$, introduce a new generator $d$ and attach an arrow labeled $d$ to any two distinct vertices in $R$. The resulting structure qualifies as the boundary of a general relator, and since it contains $R$ in its boundary it has height $k+1$. Variations on this theme can create presentations which contain general relators of arbitrary height.

As can be seen from these examples, a general relator is no longer simply a word or a cycle, but rather a particular type of simplicial complex with a labeling on its 1-skeleton. The detailed structure of a general relator is best captured by the partially ordered set of its barycenters. In each of the examples given so far we can associate a partially ordered set to each general relator. The elements are the barycenters, and the ordering is defined by setting $p<q$ if and only if $p$ is contained in the boundary of $q$. Perhaps more surprising, this partially ordered set, or poset, contains all of the information needed to reconstruct the complex. (The procedure for creating a simplicial complex from a poset is due to D. Quillen [9]). For an arbitrary partially ordered set the corresponding simplicial complex is derived by taking the finite chains as the simplices. For a traditional relator, the poset has only 3 levels: the vertices are the elements of height 0, the open edges are the elements of height 1 , and the single open 2-cell is the unique element of height 2. The simplicial complex corresponding to this poset is a simplicial subdivision of the original 2-cell. The $k$-skeleton of such a poset will refer to the geometric realization of the sub-poset consisting only of those elements of height $k$ or less. Thus in the traditional case, the 1-skeleton is the cyclic boundary and the 0-skeleton consists of the vertices alone. For the general 

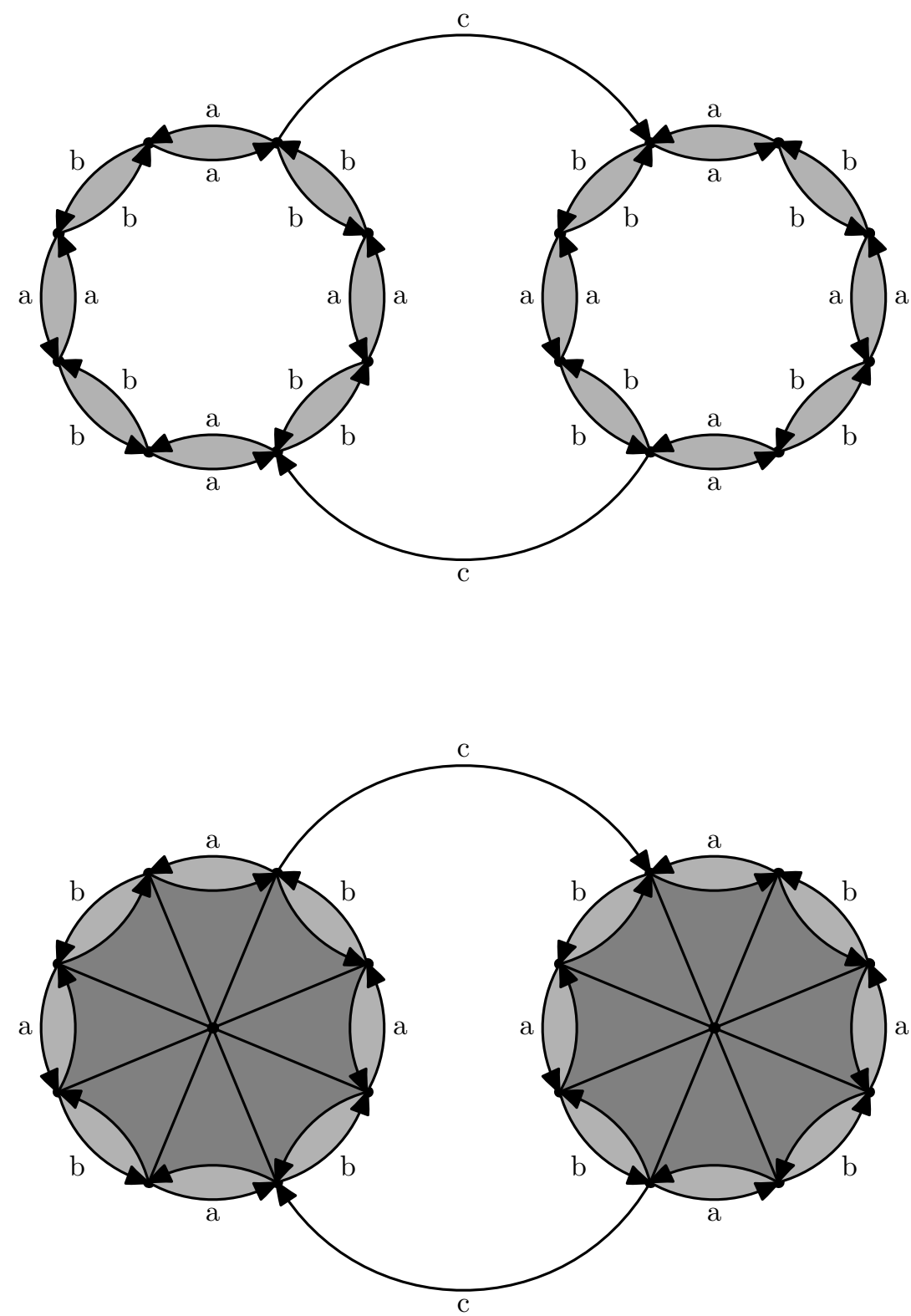

Figure 4: The 2- and 3-skeletons of a general relator of height 4 
relator shown in Figure 4 , the poset has one element of height 4, two elements of height 3 , sixteen elements of height 2 , and sixteen elements of height 1 .

We will now precisely define a general relator using these partially ordered sets.

Definition 2.7 [General Relators] A general relator $R$ is (the simplicial complex corresponding to) a finite poset with a unique maximum element subject to certain additional restrictions. First, the 1-skeleton of the poset must be a simplicial subdivision of a graph, and the unsubdivided graph needs to be deterministically labeled by a set of generators. Next, for all elements $p$ of height at least 2, the geometric realization of the ideal of all elements strictly below $p$ must be homotopically equivalent to a circle. In other words, the simplicial complex corresponding to this ideal should be homotopically equivalent to the space $S^{1}$. If the unique maximum element has height $k$ then the general relator $R$ is said to be of height $k$, and the $(k-1)$-skeleton of $R$ is called its boundary. General relators were introduced by the author in [5].

Notice that the boundary of a general relator is allowed to be fairly complicated as long as it is homotopically equivalent to the unit circle. Although a general relator can contain a 1-skeleton which can be significantly more complicated than is allowed in the traditional theory, the local structure is often less important than its global topology. One example of a global topological concept which plays a key role in the theory is that of the winding number of a loop. A winding number is definable in this context because of the homotopic equivalence to the unit circle. A loop in the boundary of a general relator with winding number 1 will be called a representative of the general relator. Using representatives, it is possible to define more or less traditional van Kampen diagrams over collections of general relators by requiring that the label of every 2-cell in the planar van Kampen diagram be the label of a representative loop in the boundary of some general relator.

Definition 2.8 [Representatives] A representative of a general relator $R$ is any path in $R$ which forms a loop with winding number \pm 1 . There are no other restrictions on representatives: they can complete five clockwise rotations followed by four counterclockwise ones, they can self-intersect, they can even be unreduced in the free group. The main use to which these representatives will be put is to define a van Kampen diagram over a set of general relators. 
Definition 2.9 [Van Kampen Diagrams] A van Kampen diagram over a set $\mathcal{R}$ of general relators is exactly like a van Kampen diagram over a set of traditional relators except that the boundary cycle of each 2-cell in the diagram is a path which maps onto a representative path in one of the general relators in $\mathcal{R}$, instead of being a relator itself.

In traditional small cancellation theory van Kampen diagrams are reduced and cancellable pairs are removed until in the reduced diagram a curvature condition forces a large portion of a relator to exist on the boundary. In the general theory described here a similar strategy is pursued, but one situation arises which does not need to be considered in the traditional case.

Example 2.10 [Self-bordering Cells] The annular diagram shown in Figure $\mathrm{O}$ is a van Kampen diagram over the presentation given in Figure 2 . More explicitly, starting at the rightmost vertex, and reading counterclockwise around the outer circle, the boundary of the 2-cell in the diagram reads the word $W=a b a b a^{-1} b^{-1} a^{-1} b^{-1}$, and this is a path which is a representative of the rightmost relator in Figure 2. The difficulty that this diagram causes is that the word bab is long relative to the length of the boundary. In the traditional theory, an overlap longer than a piece is prohibited by the fact that no (nontrivial) word in the free group is conjugate to its own inverse. The word $W$, however, is conjugate to its own inverse, as Figure 2 demonstrates.

When diagrams such as this are encountered as subdiagrams during the reduction of a van Kampen diagram, there must be some way to remove them since the goal of the reductions is to produce a reduced diagram with no long internal arcs. For Example 2.10 the reduction is easy since the entire annular subdiagram can be removed and the inner and the outer loops labeled $a$ can be identified. (The general situation is more complicated but those details will be glossed over here.)

There is another way to explain the existence of self-bordering cells in the general case. Notice that corresponding to a self-bordering 2-cell which represents a general relator $R$ there is a label-preserving automorphism of the boundary of $R$ which reverses the orientation of its boundary. In particular, the self-bordering 2-cell in Figure 5 corresponds to the automorphism of Figure 8 which rotates the left- and right-hand $a^{2}$ loops by 180 degrees. This automorphism will preserve the labels on the edges but it will reverse the orientation of the circular boundary. Traditional relators cannot have automorphisms of this type. 


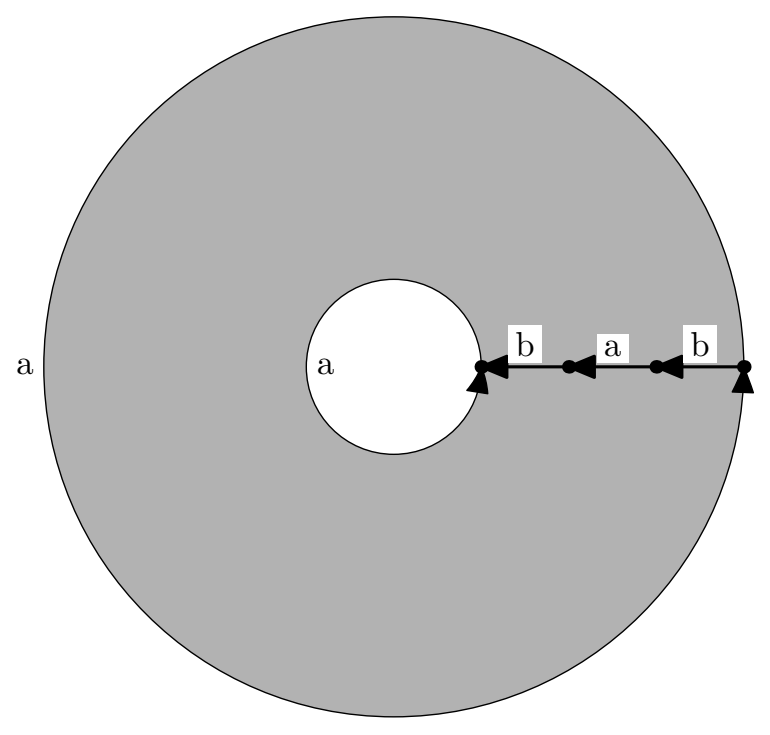

Figure 5: A 2-cell with a large overlap

Although general relators can have automorphisms which are more complicated than traditional relators, the structure of the possible automorphism groups is fairly restricted by group theoretic standards. Before the relevant theorem is quoted, however, it is necessary to make plain the meaning of two of the expressions used in its statement. First, the phrase 'closed under subcones' is just another way of stipulating that a general relator which is contained in the boundary of a general relator in the set $\mathcal{R}$ must also be contained in $\mathcal{R}$. Thus a set of general relators which contains the one whose boundary is shown in Figure 1 must also contain the general relator shown in Figure 3.

Next, a crucial cone in the boundary of a general relator $R$ is, loosely speaking, an edge or a general relator whose removal from the boundary of $R$ makes it impossible to complete a representative. In a traditional relator all of its edges are crucial. In the general case, there are some additional restrictions on the way in which the crucial cone is situated in the boundary. See [5] for details.

Theorem 2.11 ( [5]) Let $\mathcal{R}$ be a set of general relators closed under subcones and suppose that all general relators in $\mathcal{R}$ have at least one crucial cone in their boundary. If $H$ is a subgroup of the automorphism group of a general 
relator $R \in \mathcal{R}$, then for some $r$ there is a group homomorphism from $H$ to $D_{2 r}$ whose kernel is a 2-group. In particular, $H$ is isomorphic to a cyclic or a dihedral group extended by a 2-group.

The main advantage which general relators present over the traditional type is that certain symmetries which exist in the group can be captured by the relations. This obviates the need to break these symmetries by making arbitrary choices, such as selecting one representative loop of length 8 in the final relator in Figure 2 and not any of the others. When these symmetries are incorporated into the general relators themselves, the structure of the group beyond the relator level becomes more visible.

\subsection{Length and Width}

Because general relators are allowed to have boundaries which are 'thick', the portion of the boundary traversed by a path requires a bit of exposition. Along the way, a length and a width will be prescribed for each general relator. A single example will be sufficient to illustrate the nuances of these definitions.

Example 2.12 [Twisted Boundaries] Consider the presentation

$$
G=\left\langle a, b, c \mid(a b)^{3}=(b c)^{3}=(c a)^{3}=W=1\right\rangle
$$

where $W=b a b c b a^{-1} c^{-1}$. If the relator $W$ had been excluded, the presentation would satisfy $C(6)$. The relator $W$ can be altered to form a general relator by attaching a relator to $W$ whenever the cycle $W$ contains a sizable portion of this other relator. This process quickly stops at a structure which is a Möbius strip. The result is shown in Figure 6 except that the arrow on the far left labeled $a$ must be glued (in an orientation-preserving way) to the arrow on the far right labeled $a$. The word $W$ can be traced in the diagram from the upper left to the lower righthand corner. Notice also that this path becomes a loop under the gluing operation.

The resulting structure satisfies all of the conditions necessary to qualify as the boundary of a general relator. Let $R$ be the name of the general relator which results. The general relator $R$ is technically the topological cone over the Möbius strip, the Möbius strip being merely the boundary of $R$. The twisting of the boundary which occurs in this example occurs in a great many general small cancellation presentations, including those constructed for the Burnside groups. 


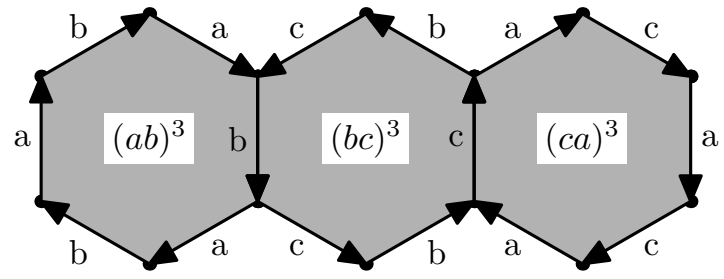

Figure 6: A construction based on the word $W$

The general relator $R$ decribed above will now be used to illustrate several key definitions. The first such concept is that of length of a general relator. For a traditional relator, the length of its boundary is simply the length of the unique reduced loop of winding number 1 . In the general case the situation is more subtle. In Example 2.12 the loop $W$ is the shortest representative of general relator $R$ and it has length 7 . But among those loops with winding number 2, some have length 12 . For loops with extremely large winding number the geodesic length of the path is more closely approximated by six times the winding number rather than by seven. In order to accurately estimate large geodesic distances, the length of a general relator must be based on the smallest average length per winding number. Thus the length of the general relator $R$ will be defined to be 6 even though there is no representative loop with that length.

Definition 2.13 [Length of a General Relator] The length of a general relator $R$ is defined to be the smallest value of the length of a loop in $R$ divided by its nonzero winding number. It is easy to show that such a minimum value must exist, and to create examples where the length of the relator is a fraction instead of an integer. The length of $R$ will be denoted $|R|$. Finally, notice that for traditional relators, this procedure gives the traditional value for the length.

Now that the length of the relator itself has been clarified, it is possible to define the relative length of a path in the boundary of a general relator.

Definition 2.14 [Graph Metric] The (normalized) graph metric for a general relator $R$ is a function which assigns a nonnegative length to every path in the boundary of $R$. In particular, if $U$ is a path in $R$ whose lift to the universal cover of the boundary of $R$ is a geodesic path, then the length of 
$U$ relative to $R$, denoted $|U|_{R}$, is the length of $U$ divided by the length of $R$. If the path $U$ is not a geodesic when it is lifted to the universal cover of the boundary of $R$, then let $V$ be a geodesic path in the universal cover between the same vertices as $U$ and set $|U|_{R}$ equal to $|V| /|R|$. Equivalently, the normalized length of $U$ is the length of the shortest path which is homotopic to $U$ in the boundary of $R$ relative to its endpoints, divided by the length of $R$ defined above.

Consider the path $W$ in the relator $R$ from Example 2.12. Since the length of the general relator $R$ is 6 , since the length of the word $W$ is 7 , and since $W$ is a geodesic when lifted to the universal cover of the boundary of $R$, $|W|_{R}=\frac{7}{6}$. A metric similar (and possibly identical) to the one just described will be used to test whether a set of general relators satisfies a general small cancelation condition.

An additional concept to be illustrated by Example 2.12 is that of width. Let $R^{\infty}$ represent the universal cover of the boundary of a general relator $R$. Since the boundary of $R$ is by definition topologically equivalent to a circle, it seems clear that the universal cover should look topologically like the real line. Thus $R^{\infty}$ should have two infinite 'ends', one going in the positive direction and the other going in the negative. These intuitions are true and they can be made precise but we will not do so here. See [0] for further details.

The width of a general relator is the smallest integer needed to guarantee that the infinite ends of $R^{\infty}$ will be disconnected from each other upon the removal of an open ball, regardless of the vertex used for the center.

Definition 2.15 [Width of a General Relator] The width of a general relator $R$, denoted $\omega_{R}$, is the unique smallest integer needed to guarantee that the removal of an open ball of radius $\omega_{R}$ centered at an arbitrary vertex in $R^{\infty}$ will always disconnect the two infinite ends. Technically, only the 1-skeleton must be disconnected; the higher-dimensional skeleta are ignored. Also, for convenience, an open ball will be defined so that it always contains the vertex used as its center. As a result, the width of a traditional relator is 0 .

In Example 2.12, the width of the general relator $R$ is 3 . To see this, consider the vertex directly below the label $(b c)^{3}$ in Figure 6, and call this vertex $u$. Assume in addition that the figure is a portion of the universal cover, so 
that the row of adjacent hexagons extends infinitely in both directions. An open ball of radius 1 centered at $u$ will remove only the vertex $u$ and the two open edges incident with it. An open ball of radius 2 will remove a total of six edges and three vertices, but a path running along the top edge of the structure still succeeds in connecting the ends. With a radius of 3 , the ends are disconnected, and the three connected components which result are the positive end, the negative end, and an isolated vertex which is directly above the vertex $u$.

\subsection{General Presentations}

A general presentation is a set of generators $A$ together with a collection of general relators $\mathcal{R}$ which are labeled by $A$. Such a presentation will be denoted $G=\langle A \mid \mathcal{R}\rangle$. The group assigned to a particular general presentation is based on the fundamental group of a topological space constructed from the generators and relators. For traditional presentations the standard construction of a 2-complex has the described group as its fundamental group. This complex is sometimes referred to as Poincaré's construction. For a general presentation there is a variation on this procedure. The resulting complex will be called the Poincaré construction of the presentation.

Definition 2.16 [Poincaré Constructions] The Poincaré construction of a general presentation is formed by taking a single vertex, attaching an edge for each of the generators, and then attaching the general relators of successively higher height, in order. The attaching of the general relators of height 2 yields a structure that is precisely the standard 2-complex corresponding to these generators and relations. If the set of general relators, $\mathcal{R}$, is closed under subcones and the relators are attached in order of increasing height, then when a relator $R$ is attached, all the edges and general relators used to construct the boundary of $R$ have already been added to the Poincaré construction. In addition there exists a unique map from the boundary of $R$ into the structure as constructed so far. The general relator $R$ is then attached using the map from the boundary to the developing structure as the attaching map. After all the relators have been added, the result is the completed Poincaré construction.

Example 2.17 The idea behind this construction can be illustrated using Example 2.2. Of the three general relators in this presentation, two are 
traditional relators of height 2 and the final relator is of height 3 . The Poincaré construction for this presentation begins by attaching the disks labeled $a^{2}$ and $b^{2}$ to a bouquet of two circles labeled $a$ and $b$. The construction at this point is the standard 2-complex whose fundamental group is the infinite dihedral group. The final general relator is now attached along its boundary to the construction. Its boundary is illustrated in Figure 2. The relator itself is shown in Figure 3. Once attached the new complex will have the dihedral group of order 8 as its fundamental group.

Definition 2.18 [Cayley Categories] The Cayley category of a presentation is based on the universal cover of the Poincare construction, but the duplication which arises in the universal cover is removed. The details are as follows. The universal cover of the Poincaré construction contains a 1skeleton which is the Cayley graph of this group. As in the traditional theory, the universal cover of the Poincaré construction may contain multiple copies of a general relator attached to this Cayley graph by functions which agree on the 1-skeletons in their boundaries. This occurs precisely when the general relator under consideration possesses nontrivial automorphisms. The number of such multiplicities is governed by the size of the automorphism group of the particular general relator involved. Once these multiple copies are eliminated through a suitable identification process, the resulting structure is called the Cayley category of the presentation. The map from the universal cover to the Poincaré construction factors through the Cayley category.

Example 2.19 If we again use the dihedral group of order 8 as an example, the universal cover of the Poincaré construction has the Cayley graph as its 1-skeleton, and has two disks labeled $a^{2}$ attached to each $a^{2}$ loop, and two disks labeled $b^{2}$ attached to each $b^{2}$ loop. If we forget the way they are mapped into the Poincaré construction, there is a natural way to identify two disks which are already attached along their boundaries. Once we perform these identifications, we next look at the 3-skeleton. We now have eight copies of Figure 3 attached to the 2-skeleton, one for each of the distinct label-preserving automorphisms of its boundary. Again, we ignore the eight distinct ways in which these are mapped to the Poincaré construction and simply identify the interiors. The result in this case is the structure shown in Figure 3. In general the Cayley category is infinite. This example is a special case since the relator contains the entire Cayley graph in its 1-skeleton. 
The height of a general relator defines a filtration on any set of general relators that is sufficient for the above constructions, but it is a filtration which is often not fine enough for more involved operations. For this reason a rank function is allowed which provides an alternative way to order the general relators in a presentation.

Definition 2.20 [Rank of a Relator] A rank function on a presentation $G=\langle A \mid \mathcal{R}\rangle$ is a function from $A \cup \mathcal{R}$ to the natural numbers which assigns each generator a rank of 1 , and all of the general relators in $\mathcal{R}$ a rank of at least 2 . The only restriction is that if $R$ is a general relator which is contained in the boundary of the general relator $S$, then $\operatorname{rank}(R)<\operatorname{rank}(S)$.

\section{General Small Cancellation Theory}

A very brief overview of general small cancellation theory will now be given. The reader is referred to [5] for further details and for the definition of any concepts which are not defined here. A general small cancellation presentation of a group $G$ is a measured presentation $G=\langle A \mid \mathcal{R}\rangle$ which satisfies the axioms of general small cancellation theory. The presentation is measured in the sense that each of the general relators in the set $\mathcal{R}$ is required to possess a function which measures the length of paths in the boundary of the relator for the purposes of the small cancellation conditions. Following a discussion of these metrics, the axioms of general small cancellation theory will be discussed in detail. The section concludes with a statement of the results derived in [5].

\subsection{Relator Metrics}

To generalize the small cancellation hypotheses to the context of general relators requires the introduction of functions which measure the extent to which a particular path wraps around the boundary of a general relator. The function on paths in $R$ will be called the relator metric for $R$ and it will be denoted $d_{R}$. The use of the term 'metric' is justified by the fact that the function $d_{R}$ will induce a metric on the points in the universal cover of the boundary of $R$. The exact properties required of the function $d_{R}$ are listed below. 
Definition 3.1 [Relator Metrics] A relator metric for a general relator $R$ is a function $d_{R}$ which assigns a nonnegative real number to every path in $R$. The function $d_{R}$ must also satisfy the following six properties:

1. $d_{R}(U)=d_{R}(V)$ whenever $U V^{-1}$ is a contractible loop in $\partial R$

2. $d_{R}(U) \geq 0$ and $d_{R}(U)=0$ iff $U$ is a contractible loop in $\partial R$

3. $d_{R}(U)=d_{R}\left(U^{-1}\right)$

4. $d_{R}(U V) \leq d_{R}(U)+d_{R}(V)$

5. if $U$ is a path which forms a loop in $\partial R$ then $d_{R}(U) \geq$ wind.num. $(U)$

6. if $U$ and $V$ are paths in $R$ which differ by an automorphism of $R$ then $d_{R}(U)=d_{R}(V)$.

One example of a relator metric which is always available is the normalized graph metric defined in the previous section. Although the graph metric always satisfies the definition, the added flexibility of allowing other metrics will be retained since other possibilities prove to be useful in the construction of the general small cancellation presentation for the Burnside groups. A general relator together with a specified relator metric is said to be measured. Similarly, a general presentation $G=\langle A \mid \mathcal{R}\rangle$ over a set of measured relators is called a measured presentation.

Definition 3.2 [Reduced Words] Corresponding to the various ways of measuring the length of a path, there are several ways of describing the 'tautness' of a word $W$. Most familiarly, a geodesic is a word which is not equivalent to any strictly shorter word in $G$. The word $W$ will be called Dehn-reduced if there do not exist words $U$ and $V$ such that $U$ is a subword of $W, U V$ is readable as a loop in one of the general relators in $\mathcal{R}$, and the length of $V$ is strictly less than the length of $V$. As in the traditional small cancellation theory, a geodesic is always Dehn-reduced but the converse is false. Finally, $W$ is said to be $\mu$-free if it is reduced in the free group and does not contain more than $\mu$ of a relator in $\mathcal{R}$ as measured by its relator metric. Specifically, there cannot exist a word $U$ and a relator $R$ such that $U$ is a subword of $W$ as well as a path in $R$ and $d_{R}(U)>\mu$. 
The multiplicity of metrics and the variety of corresponding reductions arise from various facets implicit in small cancellation theory. For instance, the curvature condition on van Kampen diagrams arises from the purely combinatorial and decidedly nonmetric condition $C(6)$, while the strict shortening of the length of a word is necessary in many cases to guarantee that a process will stop in a finite number of steps. As long as the metrics are suitably related to each other, different metrics can fulfill these two roles. In the present system these roles will be performed by the relator metrics and the (normalized) graph metrics, respectively.

\subsection{The Axioms}

This particular version of general cancellation theory requires seven axioms involving five constants. While the numbers may seem large, the axioms are far from independent, as are the constants. The redundancy built into the system is intended to improve the overall conceptual clarity. After the constants and the axioms are described informally, the exact statements will be given.

The five constants $(\alpha, \beta, \gamma, \delta$, and $\epsilon)$ are functionally defined in the first five axioms. Of these, the constant $\alpha$ is the most analogous to the constant used in traditional small cancellation theory in that it measures the degree to which one relator can be contained in another without being subsumed. Specifically, the first axiom states that if $U$ is a path which can be found in two distinct general relators (or in one general relator in two non-isomorphic ways) then either the measure of $U$ in the relator metrics is small (less than $\alpha$ ) or else the general relator $R$ for which $d_{R}(U) \geq \alpha$ is contained inside the boundary of the other relator. This can be said more precisely using maps. The second axiom states that if a lower ranked relator overlaps with a higher ranked relator then any path contained in the overlap is small $(<\beta)$ when measured by the relator metric for the higher ranked relator. The situation described in the second axiom is in some sense redundant since whenever the first axiom holds for some $\alpha$, the second axiom holds for $\beta$ equal to the same constant.

Axiom three specifies that the ratio of the width of a general relator to its length must be at most $\gamma$. The fourth states that the value given by the relator metric $d_{R}$ never differs from the value given by the normalized graph metric $|\cdot|_{R}$ by more than $\delta$. The fifth is probably the least used. If $U$ is the shortest path from a vertex in $R$ to a loop in $R$ with a non-trivial winding 
number, then the measure of $U$ using the relator metric is at most $\epsilon$. The seventh axiom describes a few convenient relationships between the constants described above. The only constraints which have been included are those needed to derive the most basic results for general small cancellation theory. Other restrictions on the constants will be listed in the statements of the theorems as they are needed.

The only axiom which has not yet been described is Axiom 6. There is a situation which arises in the reduction of a van Kampen diagram to a reduced form in which a 2-cell in the diagram overlaps with itself to form an annular subdiagram. In traditional small cancellation theory the overlapping path can contain at most a subword of a piece since anything longer would imply that a word in the free group is conjugate in the free group to its own inverse, and this is a contradiction. In the case of a representative loop of a general relator, however, such a long overlap is possible, and the sixth axiom simply states that should this situation arise, there is always a way to reduce the diagram further. Figure 5 shows an example of how this can occur.

Definition 3.3 [The Axioms] The axioms of general small cancellation theory are as follows:

Axiom 1 There is a constant $\alpha$ such that every general relator $R \in \mathcal{R}$ is $\alpha$-closed with respect to $\mathcal{R}$. In particular, if $U$ is a word readable in general relators $R$ and $S$ via $\mathcal{R}$-functors $f$ and $g$ respectively, and $d_{R}(U) \geq \alpha$, then, since $S$ is $\alpha$-closed, there exists a unique $\mathcal{R}$-functor $h: R \rightarrow S$ such that $h f=g$.

Axiom 2 There is a constant $\beta$ such that whenever a word $U$ is readable in general relators $R, S \in \mathcal{R}$ by $\mathcal{R}$-functors $f$ and $g$ respectively, and either $\operatorname{rank}(R)<\operatorname{rank}(S)$ or $\operatorname{rank}(R)=\operatorname{rank}(S)$ but there does not exist an $\mathcal{R}$ functor $h: R \rightarrow S$ with $h f=g$, then $d_{S}(U)<\beta$.

Axiom 3 There is a constant $\gamma$ such that for all general relators $R \in \mathcal{R}$, $\omega_{R} \leq \gamma|R|$.

Axiom 4 There is a constant $\delta$ such that the length of a path $U$ in a general relator $R \in \mathcal{R}$ in the relator metric $d_{R}$ is within $\delta$ of its length in the normalized graph metric on the boundary of $R$. Specifically $\left.\left|d_{R}(U)-\right| U\right|_{R} \mid \leq \delta$. 
Axiom 5 There is a constant $\epsilon$ such that whenever $U$ is the shortest possible path from a vertex in a general relator $R \in \mathcal{R}$ to a loop with nonzero winding number in $R$, the length of $U$ in the relator metric is at most $\epsilon$. That is, $d_{R}(U) \leq \epsilon$.

Axiom 6 If $W=X U Y U^{-1}$ is a representative of a general relator $R$ in which both instances of $U$ are properly oriented with respect to $W$, and $d_{R}(U) \geq \alpha$, then there exists a word $V$ such that the cycle $X V Y V^{-1}$ is readable as a contractible loop in $\partial R$ extending the reading of $X$ given by $W$. The cycle thus bounds a connected and simply connected $\mathcal{R}$-diagram $\Delta$ with $\operatorname{rank}(\Delta)<\operatorname{rank}(R)$.

Axiom 7 The constants $\alpha, \beta, \gamma, \delta$, and $\epsilon$ satisfy the following constraints: $\beta \leq \alpha$, and $\gamma, \delta, \epsilon<\alpha$, and $2 \gamma+\delta \leq \alpha \leq \frac{1}{6}$.

A general small cancellation presentation $G=\langle A \mid \mathcal{R}\rangle$ is a measured presentation which satisfies the axioms listed above. A group which possesses a general small cancellation presentation is called a general small cancellation group. The key example of an interesting general small cancellation group is, of course, the Burnside groups. In [6], a general small cancellation presentation is constructed for each of the free Burnside groups with exponent greater than or equal to 1260. The details of the construction of this general presentation are discussed in section 4 .

\subsection{The Results}

Once the proper definitions are in place the proofs of the usual small cancellation results are slight variations on the traditional proofs. It thus follows quickly that subject to a few restrictions on the values of the constants, the word problem and the conjugacy problem are decidable.

Theorem 3.4 (汭) If $G=\langle A \mid \mathcal{R}\rangle$ is a general small cancellation presentation with $3 \alpha+2 \gamma+\delta \leq \frac{1}{2}$, then the group $G$ has a decidable word problem. In particular, these results are true when $\alpha \leq \frac{1}{6}$ and $\gamma=\delta=0$ or whenever $\alpha \leq \frac{1}{8}$. 
Theorem 3.5 ( $[5])$ If $G=\langle A \mid \mathcal{R}\rangle$ is a general small cancellation presentation with $4 \alpha+2 \gamma+\delta \leq \frac{1}{2}$, then the group $G$ has a decidable conjugacy problem. In particular, this is true when $\alpha \leq \frac{1}{8}$ and $\gamma=\delta=0$ or whenever $\alpha \leq \frac{1}{10}$.

Similarly, a finitely presented general small cancellation presentation is word-hyperbolic. The proof of this particular theorem uses small cancellation conditions to prove that these groups satisfy Rip's thin triangle conditon.

Theorem 3.6 ( $[5])$ If $G=\langle A \mid \mathcal{R}\rangle$ is a finitely presented general small cancellation presentation with $3 \alpha+\delta \leq \frac{1}{3}$ and $\alpha \leq \frac{1}{10}$, then the group $G$ is word hyperbolic. In particular, the result is true when $\alpha=\frac{1}{10}$ and $\gamma=\delta=0$, or whenever $\alpha \leq \frac{1}{12}$.

In the traditional theory, the Cayley complex of a small cancellation presentation is shown to be aspherical. The following is the general small cancellation version of this fact.

Theorem 3.7 (汭) If $G=\langle A \mid \mathcal{R}\rangle$ is a general small cancellation presentation, and $C$ is the Cayley category of the presentation, then $C$ is contractible.

As discussed earlier, the difference between the universal cover of the Poincaré construction and the Cayley category of a general presentation is determined by the automorphism groups of the relators. As a result, presentations whose relators have no nontrivial automorphisms have additional properties.

Theorem 3.8 ( $\llbracket 5])$ If $G=\langle A \mid \mathcal{R}\rangle$ is a finitely presented general small cancellation presentation with $\alpha \leq \frac{1}{10}$, then the following five conditions are equivalent:

(1) the group $G$ is torsion-free

(2) all of the general relators in $\mathcal{R}$ have no nontrivial automorphisms

(3) the universal cover of the Poincaré construction is collapsed

(4) the universal cover of the Poincaré construction is contractible

(5) the Poincaré construction is a $K(G, 1)$-space.

The final result of general small cancellation theory concerns the relationship between the automorphism groups of the general relators in a presentation and the finite subgroups of the group described by the presentation. Under fairly mild restrictions the two lists are identical. 
Theorem 3.9 (汭) If $G=\langle A \mid \mathcal{R}\rangle$ is a general small cancellation presentation with $2 \beta+2 \gamma+\delta \leq \alpha \leq \frac{1}{12}$ in which $\operatorname{str}(W)$ is finite and effectively constructible for all words $W \in A^{*}$, and such that all general relators in $\mathcal{R}$ have at least one crucial cone in their boundary, then every finite subgroup of $G$ is a subgroup of the automorphism group of some general relator in $\mathcal{R}$.

Since the automorphism groups of general relators possessing crucial cones were described in Theorem 2.11, the following corollary is immediate.

Corollary 3.10 ([5]) If $G=\langle A \mid \mathcal{R}\rangle$ is a general small cancellation presentation with $2 \beta+2 \gamma+\delta \leq \alpha \leq \frac{1}{12}$ in which $\operatorname{str}(W)$ is finite and effectively constructible for all words $W \in A^{*}$, and such that all general relators in $\mathcal{R}$ have at least one crucial cone in their boundary, then each finite subgroup of $G$ can be embedded in the extension of a dihedral group by a 2-group. As a consequence, a general small cancellation group (satisfying the above conditions) which does not contain any elements of even order has only cyclic finite subgroups.

\section{Burnside Groups}

Before discussing the results concerning the Burnside groups in greater detail, the reader is reminded that the descriptions given in this final section are of a preliminary nature. In particular, the exponent 1260 is especially provisional.

In the tradition of Adian, Novikov, Ol'shanskii, and Ivanov, the general small cancellation presentation of the Burnside groups, $\mathcal{B}(m, n)=\langle A \mid \mathcal{R}\rangle$, is constructed inductively. In particular, most of the lemmas in [6] are proved by simultaneous induction on a single parameter $k$. The parameter $k$ refers

to the ranks of the general relators used in the presentation. The notations used are as follows: $\mathcal{R}_{k}$ represents the set of general relators of rank $k, \mathcal{R}(k)$ represents the set of general relators of rank at most $k$, and $\mathcal{R}$ is the union of the sets $\mathcal{R}_{k}$ over all positive integers $k$. There is a corresponding list of groups: $G(k)=\langle A \mid \mathcal{R}(k)\rangle$, and $G=\langle A \mid \mathcal{R}\rangle$. After a few of the details of the inductive construction are presented, the conclusions which result from the existence of a general small cancellation presentation for the Burnside groups will be listed. We begin with a simple illustration of the approach.

Example 4.1 [The Main Idea] Consider a presentation of the form $G=$ $\left\langle A \mid X^{n}, Y^{n}\right\rangle$ where $n$ is large and $X$ and $Y$ are simple words. It is well-known 


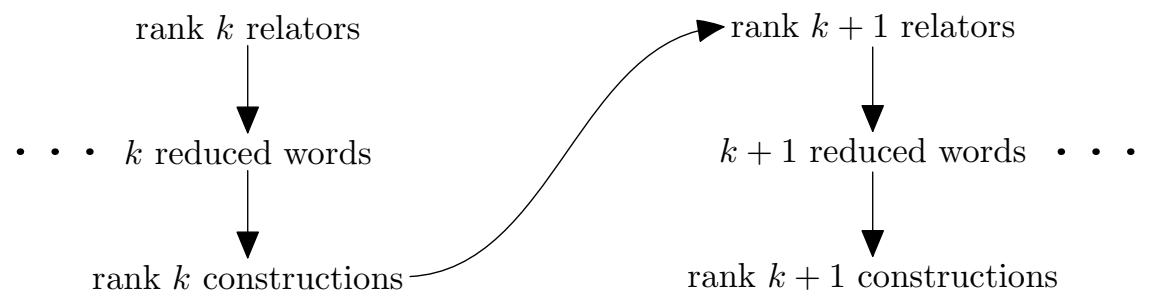

Figure 7: The order of definition

that the only way that a high power of $X$ can occur as a subword of $Y^{n}$ is if the length of $X$ is small compared to that of $Y$. More precisely, if $X^{i}$ is a subword of $Y^{n}$ then it is also a subword of a conjugate of $Y^{2}$. In this case, the construction described in outline below will attach disks with boundaries labeled $X^{n}$ to each of the subwords $X^{i}$ in $Y^{n}$. The result will be somewhat similar to the general relator shown in Figure 3 except that that general relator corresponds to the word $(a b c b)^{2}$ instead of a higher power. In general, we will begin with a word $Y^{n}$ and then we will attach previously constructed general relators whenever they have a high power of their defining word contained in the general relator under construction. Under appropriate restrictions, this process of attaching general relators stops, and the result has sufficiently nice properties to be of use in an inductive construction.

\subsection{The Inductive Construction}

The general relators used to present the Burnside groups are defined one rank at a time. The rank, $k$, indexes not only a set of general relators, but also a set of words, a set of cycles, and a set of constructions. The definitions of these concepts are intertwined and inductively defined. The cycle of definitions is as follows: reduced words and cycles in rank $k$ are used to construct the rank $k$ straightline and circular constructions. These constructions are then used to define the rank $k+1$ general relators, which are in turn used to define reduced words and cycles in rank $k+1$. See Figure 7 .

Before describing the induction itself in more detail, a word should be said about the values of the constants used in the inductive construction and about the definitions in low cases. The values of the constants used are as follows: 


$$
\alpha=\frac{1}{12}, \beta=\frac{1}{210}, \gamma=\frac{1}{70}, \delta=\frac{1}{30}, \epsilon=\frac{1}{630}, n \geq 1260
$$

These values are sufficient to satisfy all of the additional restrictions placed on the value of the constants in the statements of the results listed in Section 3 .

The induction starts as follows. Since by definition all general relators have a rank of at least 2 , there are no rank 1 relators and $\mathcal{R}_{1}=\emptyset$. The cycle of definitions thus really begins with the notions of 1-reduced words and 1reduced cycles. A word (cycle) is called 1-reduced iff it is reduced (cyclically reduced) in the free group. If $W$ is a 1-reduced word, then $\operatorname{str}_{1}(W)$ is $\operatorname{simply}$ the abstract path labeled by the word $W$. Similarly, if $W$ is a 1-reduced cycle then $\operatorname{cir}_{1}(W)$ is the abstract loop labeled by the cycle $W$. The definitions are then extended to arbitrary words (respectively, cycles) which are not 1-reduced by defining the rank 1 straightline (circular) construction on the word (cycle) to be the appropriate construction of its reduction in the free group. The rank 2 general relators are defined from the rank 1 constructions. In particular, if $W$ is a simple word in the traditional sense (i.e. not equal to a proper power of a shorter word), and if the cycle of $W$ does not contain $\beta n$ powers of any simple word, then the cone over the construction $\operatorname{cir}_{1}\left(W^{n}\right)$ is a rank 2 general relator. The set of all rank 2 general relators is called $\mathcal{R}_{2}$. Since these relators do not contain any $\beta n$ powers of any simple word, they will not contain $\beta$ of the boundary of any of the other rank 2 relators. Thus the rank 2 relators satisfy the traditional small cancellation hypothesis $C^{\prime}(\beta)$. As a consequence, the group $G(2)$ is a traditional small cancellation group.

The rank 1 straightline and circular constructions have already been defined. The rank 2 versions of these constructions will be illustrated using Figure 6. Let $G=\left\langle a, b, c \mid(a b)^{3}=(b c)^{3}=(c a)^{3}\right\rangle$, let $W=b a b c b a^{-1} c^{-1}$, and let the three relators in $G$ be assigned a rank of 2 . The construction $\operatorname{str}_{2}(W)$ is shown in Figure 6. The construction $\operatorname{cir}_{2}(W)$ is the structure obtained by gluing the left and the right edges according to orientation.

Definition 4.2 [The Straightline Construction] The rank $k$ straightline construction on a $k$-reduced word $W$ is in some sense the smallest structure which contains $W$ as a path and which is $\alpha$-closed with respect to $\mathcal{R}(k)$. The latter condition means that given any path $U$ in the structure and a general relator $R \in \mathcal{R}(k)$ such that $d_{R}(U) \geq \alpha$, the relator $R$ is already attached to the structure along this path $U$. 
Definition 4.3 [The Circular Construction] The circular construction is defined similarly. The rank $k$ circular construction on a $k$-reduced cycle $W$ is the smallest structure which contains $W$ as a loop and is $\alpha$-closed with respect to $\mathcal{R}(k)$.

If $G=\langle A \mid \mathcal{R}\rangle$ is any general small cancellation presentation with $\alpha \leq \frac{1}{8}$, it is shown in [5] that the rank $k$ straightline and circular constructions on $W$ exist and are well-defined. Under certain additional restrictions, the constructions which result are finite. The presentation constructed inductively for the Burnside groups satisfies these restrictions, and it is the rank $k$ circular constructions which are used to define the boundaries of what will become the rank $k+1$ general relators. Once these relators have been constructed, the axioms of general small cancellation are shown to hold. At that point the results listed in the previous section become available, and the induction continues.

\subsection{The Results}

The most important result on the Burnside groups of sufficiently large exponent that is established in [6] is that they possess a general small cancellation presentation.

Theorem 4.4 For $n \geq 1260$ the Burnside group $\mathcal{B}(m, n)$ possesses a general small cancellation presentation.

Most of the other results for these groups follow immediately from the existence of such a presentation, and from the general small cancellation theory described in the previous section. In particular, the following corollary is immediate from Theorem 3.4 and Theorem 3.5 .

Corollary 4.5 For all $n \geq 1260$, the Burnside group $\mathcal{B}(m, n)$ has a decidable word problem and a decidable conjugacy problem.

As might be expected, the classification of the structure of the finite subgroups of the Burnside groups is more specific than the structure of the finite subgroups in a general small cancellation group in general.

Theorem 4.6 Every finite subgroup of the group $\mathcal{B}(m, n)$ is contained in a direct product of a dihedral of order $2 n$ with a finite number of dihedral 2-groups whose exponent divides $n$. 
It is from the classification of the finite subgroups that the last two major results are derived.

Corollary 4.7 For all $n \geq 1260$ and all $m \geq 2$, the Burnside group $\mathcal{B}(m, n)$ is infinite.

Proof: If $\mathcal{B}(m, n)$ were finite, then it would itself be a finite subgroup. Thus, once it is shown that $\mathcal{B}(m, n)$ cannot be embedded in a finite product of dihedral groups, the proof will be complete. It is an easy matter to demonstrate this.

Corollary 4.8 For all $n \geq 1260$ and all $m \geq 2$, the Burnside group $\mathcal{B}(m, n)$ is not finitely presented.

Proof: If $\mathcal{B}(m, n)$ were finitely presented, then by Theorem 3.6 it would be a word hyperbolic group, and thus automatic. However, it is well known that finitely presented infinite torsion automatic groups do not exist. 


\section{References}

[1] S. Adian. The Burnside Problem and Identities in Groups. SpringerVerlag, New York, 1979.

[2] W. Burnside. An unsettled question in the theory of discontinuous groups. Quart. J. of Pure and Appl. Math., 33 (1902), 230-238.

[3] S. Ivanov. The free Burnside groups of sufficiently large exponents. International Journal of Algebra and Computation, 4 (1994), 1-308.

[4] I. Lysionok. Infinite Burnside groups of even period. Izv. Ross. Akad. Nauk Ser. Mat. 60 (1996), 3-224.

[5] J. McCammond. A general small cancellation theory to appear in Int. J. of Alg. and Comp.

[6] J. McCammond. The Burnside groups via a general small cancellation theory. In preparation.

[7] P. Novikov and S. Adian. On infinite periodic groups, I, II, III. Izv. Acad. Nauk. SSSR Ser. Matem., 32 (1968), 212-244,251-524,709-731.

[8] A. Yu. Ol'shanskii. On the Novikov-Adian theorem. Math. Sbornik, 118 (1982), 203-235.

[9] D. Quillen. Higher Algebraic K-Theory: I. Springer-Verlag, New York, 1973 .

[10] E. Rips. Generalized small cancellation theory and applications I. Isr. J. Math. 41 (1982), 1-146. 